\title{
Nicotine Dependence and Smoking Cessation after Hospital Discharge among Inpatients with Coronary Heart Attacks
}

\author{
Atsuhiko OTA ${ }^{1}$, Yoshio MINO$^{2}$, Hiroshi MIKOUCHI ${ }^{3}$ and Norito KAWAKAMI ${ }^{1}$ \\ ${ }^{\prime}$ Department of Hygiene and Preventive Medicine, Okayama University Graduate School of Medicine and Dentistry, Okayama \\ ${ }^{2}$ College of Social Welfare, Osaka Prefecture University, Osaka \\ ${ }^{3}$ Department of Diseases of the Circulatory System, National Okayama Medical Center, Okayama
}

\begin{abstract}
This study focuses on the predictability of two alternative questionnaires for nicotine dependence, i.e., the Fagerstrom Tolerance Questionnaire (FTQ) and the Tobacco Dependence Screener (TDS), each of which represents a different aspect of dependence, among patients with coronary heart disease (CHD). Twenty-nine male inpatients that had been newly diagnosed as CHD were followed up for 30 weeks after hospital discharge. The baseline information included age, number of cigarettes per day, years of smoking, disease type (angina pectoris [AP] or acute myocardial infarction [AMI]), whether they had received a briefing on smoking cessation, the FTQ, and the TDS. At 30 weeks after hospital discharge, $19(66 \%)$ were abstainers. The group with a high TDS score (of 6 or greater) was significantly less likely to quit smoking than the group with a low TDS score $(p=0.046)$. The FTQ score was not significantly different between the abstainers and non-abstainers. The subjects with AP were significantly less likely to quit smoking than those with AMI ( $p=0.021)$. Multiple logistic regression analysis showed that belonging to the high-TDS group and being diagnosed as AP were significantly associated with failure in smoking cessation $(p<0.05)$. The present study suggests that the TDS may have higher predictability than the FTQ concerning smoking cessation among CHD inpatients.
\end{abstract}

Key words: coronary heart disease (CHD), smoking, nicotine dependence, the Fagerstrom Tolerance Questionnaire (FTQ), the Tobacco Dependence Screener (TDS)

\section{Introduction}

Smoking cessation is beneficial, even for patients with coronary heart disease (CHD) (1-5). Patients with angina pectoris (AP) who smoke have a greater risk of later CHD events or death than those who do not smoke (6-8). A recent meta-analysis concluded that smoking cessation among patients who have had a myocardial infarction (MI) results in a significant decrease in mortality (9). However, only approximately $1 / 3$ to $1 / 2$ of smokers who have experienced an MI subsequently reduced their tobacco intake or quit smoking (10).

Nicotine dependence is a great factor preventing smoking cessation. Several questionnaires have been developed to measure nicotine dependence. The Fagerstrom Tolerance Questionnaire (FTQ) was originally developed to measure the degree of the

Received Jan. 7 2002/Accepted Mar. 42002

Reprint requests to: Atsuhiko OTA

Department of Hygiene and Preventive Medicine, Okayama University Graduate School of Medicine and Dentistry, 2-5-1 Shikata-cho, Okayama 700-8558, Japan

TEL: +81(86)235-7174, FAX: +81(86)235-7178

E-mail: a-ohta@mx1.tiki.ne.jp physiological aspects of nicotine dependence, such as cardiovascular reactions after smoking cessation $(11,12)$. The FTQ is now widely used for participants of smoking cessation therapy programs. Fagerstrom and Schneider (13) suggested in their review that the FTQ was a good predictor of outcome in a smoking cessation trial without nicotine-substitution therapy. However, only a few studies have been conducted to test the predictive ability of the FTQ among CHD patients, and the findings are conflicting. Jerome et al. (14) reported that MI patients with lower FTQ scores were more likely to have remained abstinent at a 6month follow-up than those with high FTQ scores. On the other hand, Dornelas et al. (15) reported that there was no significant relation between the FTQ score and smoking cessation among acute MI (AMI) patients. It should be further examined whether the FTQ is useful to predict successful smoking cessation.

Tobacco dependence may be a multi-dimensional contract including psychological and behavioral aspects of dependence on smoking or nicotine itself as well as physiological ones (16). This is partly supported by the poor concordance between the FTQ and nicotine dependence defined in the Diagnostic and Statistical Manual of Mental Disorders, third edition (DSM-III) $(17,18)$. While the FTQ is supposed to mainly measure the physiological aspects of nicotine dependence, psychological (i.e., feeling of 
dependence) and behavioral aspects (e.g., continuing smoking despite severe illness) are included in nicotine or tobacco dependence in standardized psychiatric diagnoses, as in the Diagnostic and Statistical Manual of Mental Disorders, third edition revised and fourth edition (DSM-III-R and DSM-IV) $(19,20)$, and the International Classification of Diseases, $10^{\text {th }}$ revision (ICD-10) (21). The Tobacco Dependence Screener (TDS) was developed to measure the psychological and behavioral aspects of nicotine dependence according to the DSM-III-R, DSM-IV, and ICD-10 (22). Although it has been reported that the TDS predicts smoking cessation to some extent (22), to date no study has addressed the predictive ability of the TDS among patients with CHD.

A randomized controlled trial study was conducted for smokers among CHD inpatients to determine the effect of a briefing as an intervention on smoking cessation after hospital discharge. We conducted a secondary analysis of the data to test whether the FTQ and the TDS had a different degree of predictability for smoking cessation among CHD patients. The primary aim of this study was to compare the association of the FTQ and the TDS with smoking cessation, controlling for the effect of the intervention (i.e., briefing). We also briefly describe the effect of the briefing on smoking cessation among CHD patients, relating it to the predictability of the FTQ and the TDS.

\section{Subjects and Methods}

\section{Subjects}

The subjects were 29 male inpatients who were current smokers and were admitted into a general hospital in Japan with a new diagnosis of AP or AMI between August, 1999 and June, 2000. Informed consent was obtained from all subjects. Of the 29 subjects, 14 had diseases diagnosed as AP; the remaining 15 had diseases diagnosed as AMI. Baseline information on smoking behavior and nicotine dependence was collected from them from two to seven days after admission. The subjects were then randomly assigned to an intervention group $(n=11)$ or a control group $(n=18)$. The assignment was based on the last digit of the subject's home phone number: even numbers were assigned to the intervention group, while odd numbers were assigned to the control group. It could be chance that the sample size was disproportionate between these two groups. The intervention group members were briefed on smoking cessation at bedside for 5 to 10 minutes by one of the authors (AO). The briefing included the following topics: smoking as a risk factor; benefits of smoking cessation; and coping with withdrawal symptoms. Control group members did not receive the briefing. At 30 weeks after hospital discharge, the subjects were interviewed by telephone to find out whether they were still not smoking. None of the 29 subjects had dropped out.

\section{Measures}

The subjects were interviewed at bedside to collect the following baseline information after admission: age, number of cigarettes per day, years of smoking, the FTQ (11), and the TDS (22) (Table 1). The FTQ is an eight-item questionnaire for nicotine dependence. The FTQ yields a score ranging from 0 to 11 , with a greater score indicating a heavier dependency. The subjects were divided into two groups: a low-FTQ group (6 or less) and a highTDS group ( 7 or greater) $(13,23)$. The TDS consisted of 10 yes-no questions and was scored according to the number of affirmative
Table 1 Demographic and smoking-related characteristics at baseline of subjects in this study*

\begin{tabular}{lccc}
\hline Variables & $\begin{array}{c}\text { All subjects } \\
(\mathrm{n}=29)\end{array}$ & $\begin{array}{c}\text { Intervention group } \\
(\mathrm{n}=11)\end{array}$ & $\begin{array}{c}\text { Control group } \\
(\mathrm{n}=18)\end{array}$ \\
\hline Age & 66.3 & 65.8 & 66.7 \\
& $(9.3)$ & $(9.7)$ & $(9.4)$ \\
Number of cigarettes & 30.2 & 30.9 & 29.7 \\
per day & $(15.6)$ & $(13.9)$ & $(16.9)$ \\
Years of smoking & 44.3 & 42.7 & 45.3 \\
& $(10.3)$ & $(12.1)$ & $(9.2)$ \\
Fagerstrom Tolerance & 6.2 & 6.2 & 6.2 \\
Questionnaire (FTQ) & $(1.4)$ & $(1.2)$ & $(1.5)$ \\
Tobacco Dependence & 5.1 & 5.6 & 4.8 \\
Screener (TDS) & $(2.2)$ & $(2.2)$ & $(2.1)$ \\
\hline
\end{tabular}

* Figures are presented as means (S.D.). There was no significant difference in any variable between the intervention group and the control group $(p>0.05)$.

answers. A TDS score of 6 or greater indicates nicotine/tobacco dependence according to the ICD-10 diagnosis, with a sensitivity of $95 \%$ and a specificity of $81 \%$ (22). The subjects were also dichotomized into a low-TDS group (5 or less) and a high-TDS group ( 6 or greater) by using the cut-off point. In response to questions on smoking history and nicotine dependence, the subjects were asked to recall their behavior or experience before having their first heart attack.

Based on their responses to telephone interviews at 30 weeks after hospital discharge, the subjects were considered to be abstainers if they had not relapsed into smoking and non-abstainers if they had.

\section{Statistical analysis}

Student's t test was used to compare the average age, number of cigarettes per day, years of smoking, FTQ score, and TDS score between the abstainers and the non-abstainers at 30 weeks after discharge. Fisher's exact test was used to investigate the relation that smoking cessation had with the type of disease (AP or AMI), the briefing on smoking cessation (whether they had received it), the FTQ group (6 or less/7 or greater), and the TDS group (5 or less/ 6 or greater). When necessary, the $t$ test was modified for unequal variances. Finally, multiple logistic regression analysis was performed controlling for covariates. A significant level of 0.05 was used for all statistical tests, and two-tailed tests were applied. SPSS 10.0 for Windows was used for the statistical calculations (24).

\section{Results}

Of the 29 subjects, $19(66 \%)$ were abstainers at 30 weeks after hospital discharge. The average TDS score was significantly lower in the abstainers than in the non-abstainers (Table 2). There was no significant difference in the FTQ score between these two groups. The subjects with AP were significantly less likely to quit smoking than those with AMI (Table 3). The group with a high TDS score (of 6 or greater) was significantly less likely to quit smoking than the group with a low TDS score. No significant difference in smoking status was observed between the high- and low-FTQ groups. The briefing was not significantly associated with smoking cessation.

The results of multiple logistic regression analysis indicated that the high-TDS group was significantly more likely to fail in 
Table 2 Comparison of demographic characteristics by smoking status at 30 weeks after hospital discharge

\begin{tabular}{lcccc}
\hline Baseline information & Abstainers & & Non-abstainers & \multirow{2}{*}{ p value* } \\
\cline { 2 - 2 } & Mean (S.D.) & & Mean (S.D.) & \\
\hline Age & $66.6(10.8)$ & & $66.6(6.2)$ & 0.917 \\
Number of cigarettes per day & $32.6(18.1)$ & & $25.5(8.3)$ & 0.158 \\
Years of smoking & $43.7(10.8)$ & & $44.7(10.3)$ & 0.811 \\
FTQ & $6.2(1.2)$ & & $6.3(1.7)$ & 0.796 \\
TDS & $4.4(2.2)$ & $6.5(1.3)$ & 0.009
\end{tabular}

* Tested by t-test. When necessary, the t-test was modified for unequal variances.

Table 3 Smoking cessation at 30 weeks after hospital discharge by disease type, briefing or lack thereof, and TDS group among inpatients with coronary heart attacks

\begin{tabular}{|c|c|c|c|}
\hline & $\mathrm{n}$ & $\begin{array}{l}\text { Number of } \\
\text { abstainers }\end{array}$ & $\mathrm{p}$ value* \\
\hline \multicolumn{4}{|l|}{ Disease type: } \\
\hline Angina pectoris (AP) & 14 & 6 & 0.021 \\
\hline Acute myocardial infarction (AMI) & 15 & 13 & \\
\hline \multicolumn{4}{|l|}{ Briefing: } \\
\hline Intervention & 11 & 8 & 0.694 \\
\hline Control & 18 & 11 & \\
\hline \multicolumn{4}{|l|}{ FTQ score: } \\
\hline Low (6 or less) & 17 & 7 & 0.449 \\
\hline High ( 7 or greater) & 12 & 3 & \\
\hline \multicolumn{4}{|l|}{ TDS score: } \\
\hline Low ( 5 or less) & 17 & 14 & 0.046 \\
\hline High ( 6 or greater) & 12 & 5 & \\
\hline
\end{tabular}

* Tested by Fisher's exact test

Table 4 Odds ratio, $95 \%$ confidence interval (CI), and p-value estimated by multiple logistic regression analysis of failure in smoking cessation at 30 weeks after hospital discharge

\begin{tabular}{lcc}
\hline Variables & Odds ratio $(95 \% \mathrm{CI})$ & Significance \\
\hline TDS score $(6 \text { or greater })^{*}$ & $15.8(1.3-191)$ & $\mathrm{p}=0.030$ \\
Disease type** $^{* *}$ & $15.1(1.3-182)$ & $\mathrm{p}=0.033$ \\
Briefing $^{* *}$ & $0.6(0.1-6.4)$ & $\mathrm{p}=0.654$
\end{tabular}

* A group with TDS scores of 6 or greater was coded as 1, 5 or less as 0 .

** Coded as $\mathrm{AP}=1, \mathrm{MI}=0$.

$* * *$ Coded as intervention group $=1$, control group $=0$.

smoking cessation after controlling for disease type and whether they had received a briefing (Table 4). Being diagnosed as having AP was also significantly associated with failure in smoking cessation. The briefing was associated with a slightly lower risk of failure in smoking cessation, but not significantly. Being in the high-TDS group and being diagnosed as having AP were significantly associated with failure in smoking cessation, even after controlling for the number of cigarettes per day and the years of smoking, in addition to these covariates, although the odds ratios and $95 \%$ confidence intervals ( $95 \%$ CIs) were very large $(\mathrm{p}<0.05$, data not shown).

These results were similar between the intervention and control groups.

\section{Discussion}

The present study showed the predictability of the TDS for smoking cessation after discharge among inpatients with CHD.
The FTQ did not predict smoking cessation in this study. The lower predictability of the FTQ is concordant with a previous report by Dornelas and coworkers (15). It appears that the FTQ has a lower predictability for smoking cessation among CHD patients than it does among participants in a smoking cessation therapy (13). However, the previous study did not focus on the reasons behind this lower predictability (15). The FTQ originally intended to measure the degree of physiological changes immediately after smoking cessation (11), which may better predict the difficulties faced by smokers shortly after quitting. CHD patients were usually not allowed to smoke for at least several days after hospital admission, in particular, while they were in the coronary care unit (CCU). If they were not in a CCU, the medical staff closely watched them so that they did not smoke. Thus, they were forced to refrain from smoking even if they experienced the withdrawal symptoms that may occur within the first several days after smoking cessation. This may have resulted in the lower predictability of the FTQ among CHD patients. Another possibility is that, having survived a heart attack, CHD patients may be highly motivated to stop smoking, and, among those who are highly motivated, the physiological aspect of nicotine dependence is not a strong barrier to smoking cessation.

On the other hand, in the present study, the TDS significantly predicted successful smoking cessation among CHD patients. The TDS was initially developed to screen cases with nicotine dependence according to the DSM-III-R, DSM-IV, and ICD-10. Thus, the TDS is regarded as a measure of the psychological and behavioral aspects of nicotine dependence (22). Such psychological and behavioral aspects of the dependence may be more important to predict successful smoking cessation among CHD patients. Psychological aspects, such as feeling dependent on tobacco, may be a better predictor than physiological dependence among CHD patients who experienced withdrawal symptoms during their stay in a CCU. Dependent behavioral patterns assessed by the TDS, such as the continuance of smoking despite severe illness and the knowledge that smoking will antagonize the disease, may be better indicators for predicting the ability to quit smoking. These may be the reasons for the present finding that the TDS is a better predictor than the FTQ.

In practice, the findings of the present study suggest that the measure of nicotine dependence depends on the characteristics of the subjects; in other words, the FTQ may not cover all aspects of smoking behavior. In a smoking cessation support program, a suitable questionnaire of nicotine dependence should be chosen carefully according to the characteristics of the patients and the medical care that they are receiving. Based on the present findings, the TDS is recommended as the best predictor of a CHD patient's ability to quit smoking. However, our estimation of the predictability of the FTQ and the TDS may not be stable because of the small sample size in the present study: we might have underestimated the predictability of the FTQ. It is necessary to confirm the difference in predicting smoking cessation between the FTQ and the TDS with a greater number of subjects. Moreover, there are only a few studies focusing on the likelihood that the FTQ and the TDS can predict the ability of CHD patients to quit smoking. Therefore, future research will be needed to replicate the present findings and reach a conclusion about the differential predictability of these two measurements.

Tobacco dependence is a complex construct, including physiological, psychological, and behavioral aspects (16). The observed 
differential predictability of the FTQ and the TDS suggests that these two questionnaires measure different dimensions of nicotine dependence. Breslau and Johnson (25) also reported that nicotine dependence, according to the DSM-III-R, was a better predictor of the depression that is associated with smoking cessation than the Fagerstrom Test for Nicotine Dependence (FTND) (26), which is a revised version of the FTQ. These findings, together with those of the present study, suggest that nicotine dependence may have two dimensions, or possibly more, each with a different underlying mechanism. Further studies are required to clarify the nature of these components of nicotine dependence measured by the FTQ and the TDS. It may also be useful to develop a comprehensive questionnaire for measuring several aspects of nicotine dependence.

In the present study, AP patients were more likely to smoke again than AMI patients. This agrees with the previous findings that AMI patients quit smoking more frequently than AP patients (27). Patients with unstable angina, which was recently regarded as an acute coronary syndrome, also showed a significant increase in the re-smoking rate (28). A possible reason is that symptoms are more severe in patients with AMI than in those with AP. AMI patients sometimes have dyspnea, unconsciousness, and other severe symptoms and signs that AP patients seldom have. Smoking cessation was strongly associated with the severity of symptoms $(27,29-32)$. The severity of the symptoms associated with AMI might serve as a stronger motivator to quit smoking.

The briefing was associated with a lower risk of failure in smoking cessation, but this association was not significant. The large range of $95 \%$ CI implies that the effects of the briefing may vary greatly among the subjects. A short briefing with no follow-up might not have a strong and long-lasting effect. When the subjects were still in the hospital, they might have been much more interested in their prognosis and daily life after discharge than in smoking cessation. Such a short introductory education may not be fully effective for smoking cessation among patients who have experienced coronary heart attacks. A more intensive follow-up smoking cessation program focusing on the psychological and behavioral aspects of nicotine dependence, which were suggested to be important factors in the present study, may be needed to support smoking cessation among CHD patients.

We analyzed the data from an intervention trial to test the differential predictability of FTQ and TDS, which made the interpretation slightly complex. The intervention showed only a weak effect on smoking cessation, and the predictability of TDS and FTQ was similar for both the intervention and control groups. Thus, it appears possible to extend our findings to CHD smokers in general. However, the predictability of the nicotine dependence measures may depend on the situation, e.g., in a smoking cessation program or in a natural setting. The predictability of FTQ and TDS should also be tested in further studies by comparing it in various smoking cessation situations.

There are several other limitations to the present study. First, there may be a recall bias in the collection of the baseline information, including the FTQ and TDS, because of retrospective assessment, which may have resulted in the poor predictability of the FTQ. Second, we interviewed the subjects by telephone to find out whether they remained abstinent at 30 weeks after hospital discharge. Although several studies have shown or confirmed the reliability of self-reporting about smoking status (33-35), a selfreported assessment of smoking cessation has greater limitations than a biological assessment. Third, the number of subjects in the present study was not large. The present findings should be replicated in a future study with a greater number of subjects, conducting a prospective assessment of baseline smoking behaviors and nicotine dependence and a biological assessment of smoking (such as the measurement of urine cotinine).

\section{References}

( 1 ) Daly L, Mulcahy R, Graham IM, Hickey N. Long term effect on mortality of stopping smoking after unstable angina and myocardial infarction. Br. Med. J. 1983; 287: 324-326.

( 2 ) Voors AA, Van Brussel BL, Plokker HW, Ernst SM, Ernst NM, Koomen EM, Tijssen JG, Vermeulen FE. Smoking and cardiac events after venous coronary bypass surgery. A 15-year follow-up study. Circulation 1996; 93: 42-47.

( 3 ) Gensini GF, Comeglio M, Colella A. Classical risk factors and emerging elements in the risk profile for coronary artery disease. Eur. Heart. J. 1998; 19 Supple A: A53-A61.

( 4 ) Taylor BV, Oudit GY, Kalman PG, Liu P. Clinical and pathophysiological effects of active and passive smoking on the cardiovascular system. Can. J. Cardiol. 1998; 14: 1129-1139.

( 5 ) Capewell S, Morrison CE, McMurray JJ. Contribution of modern cardiovascular treatment and risk factor changes to the decline in coronary heart disease mortality in Scotland between 1975 and 1994. Heart 1999; 81: 380-386.

( 6 ) Hagman M, Wilhelmsen L, Pennert K, Wedel H. Factors of importance for prognosis in men with angina pectoris derived from a random population sample. Multifactor Primary Prevention Trial, Gothenburg, Sweden. Am. J. Cardiol. 1988; 61: 530535

( 7 ) Miwa K, Fujita M, Miyagi Y. Beneficial effects of smoking cessation on the short-term prognosis for variant angina - validation

of the smoking status by urinary cotinine measurements. Int. J. Cardiol. 1994; 44: 151-156.

( 8 ) Wilhelmsen L. Effects of cessation of smoking after myocardial infarction. J. Cardiovasc. Risk 1998; 5: 173-176.

(9) Wilson K, Gibson N, Willan A, Cook D. Effect of smoking cessation on mortality after myocardial infarction: meta-analysis of cohort study. Arch. Intern. Med. 2000; 160: 939-944.

(10) Burling TA, Singleton EG, Bigelow GE, Baile WF, Gottlieb SH. Smoking following myocardial infarction: a critical review of the literature. Health Psychology 1984; 3: 83-96.

(11) Fagerstrom KO. Measuring degree of physical dependence to tobacco smoking with reference to individualization of treatment. Addictive Behaviors 1978; 3: 235-241.

(12) Lombardo TW, Hughes JR, Fross JD. Failure to support the validity of the Fagerstrom Tolerance Questionnaire as a measure of tolerance to nicotine. Addictive Behaviors 1988; 13: 87-90.

(13) Fagerstrom KO, Schneider NG. Measuring nicotine dependence: a review of the Fagerstrom Tolerance Questionnaire. Journal of Behavioral Medicine 1989; 12: 159-182.

(14) Jerome A, Bigelow GE, Burling TA, Baile WF, Gottlieb S. Inhospital predictors of post-hospital smoking outcomes following myocardial infarction. Behav. Med. Update Annu. Meet. Addend. 1984; 2: 33-35.

(15) Dornelas EA, Sampson RA, Gray JF, Waters D, Thompson PD. 
A randomized controlled trial of smoking cessation counseling after myocardial infarction. Preventive Medicine 2000; 30: 261268.

(16) Benfari RC, Ockene JK, McIntyre KM. Control of cigarette smoking from a psychological perspective. Annu. Rev. Public Health 1982; 3: 101-128.

(17) American Psychiatric Association. Diagnostic and Statistical Manual of Mental Disorders, Third Edition. Washington DC: APA, 1980.

(18) Hughes JR, Gust SW, Pechacek TF. Prevalence of tobacco dependence and withdrawal. American Journal of Psychiatry 1987; 144: 205-208.

(19) American Psychiatric Association. Diagnostic and Statistical Manual of Mental Disorders, Revised Third Edition. Washington DC: APA, 1987.

(20) American Psychiatric Association. Diagnostic and Statistical Manual of Mental Disorders, Forth Edition. Washington DC: APA, 1994.

(21) World Health Organization. The ICD-10 classification of mental and behavioral disorders: Clinical descriptions and diagnostic guidelines. Geneva: WHO, 1992.

(22) Kawakami N, Takatsuka N, Inaba S, Shimizu H. Development of a screening questionnaire for tobacco/nicotine dependence according to ICD-10, DSM-III-R, and DSM-IV. Addictive Behaviors 1999; 24: 155-166.

(23) Westman EC, Behm FM, Simel DL, Rose JE. Smoking behavior on the first day of a quit attempt predicts long-term abstinence. Arch. Intern. Med. 1997; 157: 335-340.

(24) SPSS Inc. SPSS Base 10.0J User's Guide. Chicago, IL: SPSS Inc., 1999.

(25) Breslau N, Johnson EO. Predicting smoking cessation and major depression in nicotine-dependent smokers. Am. J. Public Health 2000; 90: 1122-1127.

(26) Heatherton TF, Kozlowski LT, Frecker RC, Fagerstrom KO. The
Test for Nicotine Dependence: a revision of the Fagerstrom Tolerance Questionnaire. British Journal of Addiction 1991; 86: $1119-1127$.

(27) Ockene J, Kristeller JL, Goldberg R, Ockene I, Merriam P, Barrett S, Pekow P, Hosmer D, Gianelly R. Smoking cessation and severity of disease: the Coronary Artery Smoking Intervention Study. Health Psychology 1992; 11: 119-126.

(28) Hasdai D, Garratt KN, Grill DE, Mathew V, Lerman A, Gau GT, Holmes DR Jr. Predictors of smoking cessation after percutaneous coronary revascularization. Mayo Clin. Proc. 1998; 73: 205209.

(29) Hay DR, Turbott S. Changes in smoking habits in men under 65 years after myocardial infarction and coronary insufficiency. Br. Heart J. 1970; 32: 738-740.

(30) Wilhelmsson C, Vedin JA, Elmfeldt D, Tibblin G, Wilhelmsen L. Smoking and myocardial infarction. Lancet 1975; 1 (7904): 415-420.

(31) Baile WF, Bigelow GE, Gottlieb SH, Stitzer ML, Sacktor JD. Rapid resumption of cigarette smoking following myocardial infarction: inverse relation to MI severity. Addictive Behaviors 1982; 7: 373-380.

(32) Frid D, Ockene IS, Ockene JK, Merriam P, Goldberg R, Kristeller J, Barrett S. Severity of angiographically proven coronary artery disease predicts smoking cessation. American Journal of Preventive Medicine 1991; 7: 131-135.

(33) Luepker RV, Pallonen UE, Murray DM, Pirie PL. Validity of telephone surveys in assessing cigarette smoking in young adults. American Journal of Public Health 1989; 79: 202-204.

(34) Slattery ML, Hunt SC, French TK, Ford MH, Williams RR. Validity of cigarette smoking habits in three epidemiologic studies in Utah. Preventive Medicine 1989; 18: 11-19.

(35) Lichtenstein E, Glasgow RE. Smoking cessation: what have we learned in over the past decade. J. Clin. Consult. Psychol. 1992; 60: $518-527$. 